\title{
Observation of disclinations and optical anisotropy in a mesomorphic copolyester
}

\author{
M. R. Mackley*, F. Pinaud $\dagger$ and G. Siekmannj \\ School of Engineering and Applied Sciences, University of Sussex, Brighton, UK \\ (Received 7 August 1980; revised 30 September 1980)
}

\begin{abstract}
We report observations carried out on a thermotropic copolyester which had been polymerized by the manufacturers with the random addition of aromatic groups into the conventional poly(ethylene terephthalate) chain. At elevated temperatures the material exhibits complex anisotropic effects in the quiescent mesomorphic state which are observed to be frozen into the material at room temperature. The copolyester is sensitive to surface effects and is possibly influenced by magnetic fields. Using the latter technique we were able to orient the polymer in a manner which enabled us positively to identify within the material the existence of disclinations with strengths of \pm 1 . Finally surface replica electron microscopy revealed a morphological texture that complimented and extended that deduced from the optical studies.
\end{abstract}

\section{INTRODUCTION}

Small-molecule liquid crystals have traditionally been classified as either lyotropic or thermotropic liquid crystals and, in an analogous way, mesomorphic polymers have been divided into these categories. The lyotropic mesophase is produced by mixing the usually stiff polymer chains with a solvent above a critical concentration. The formation of a distinct mesophase was predicted by Onsager $^{1}$ and Flory ${ }^{2}$ and the poly( $p$-benzamide) systems are typical of this type of mesomorphic form (see for example Morgan, ref 3). The existence of lyotropic polymers has been known since the late 1960s and Dupont's 'Kevlar', high strength fibre is believed to be manufactured from a process utilizing the polymers' lyotropic mesophase properties.

The thermotropic mesophase which is the primary concern of this paper is produced when the polymer exists alone but within a certain temperature range which is usually above the normal solidification temperature. Recently Jackson and Kuhfuss ${ }^{4}$ produced a random copolyester polymer which exhibited properties that were consistent with thermotropic mesomorphic behaviour and this material has introduced a new class of polymer which has bulk physical and structural properties that are significantly different from both traditional crystalline and amorphous polymers. This type of material presents new opportunities of obtaining highly oriented polymer systems and the materials' unusual rheological properties can be expected to influence their future processing procedures.

The material we have studied was prepared by the Eastman Kodak Company from $40 \mathrm{~mol} \%$ poly(ethylene terephthalate) and $60 \mathrm{~mol} \% \mathrm{p}$-acetoxybenzoic acid. According to Jackson and Kuhfuss the final linear polymer consists of the following segments:

* Present address: Department of Chemical Engineering, University of Cambridge, Pembroke Street, Cambridge, UK

$\dagger$ Present address: Rhone Poulenc, Direction des Recherches et du Developpement, 11 Avenue de la Republique, 69631 Venissieux, France.

+ Present address: Institut für Werkst offe, Ruhr-Universität, Bochum,

W. Germany.

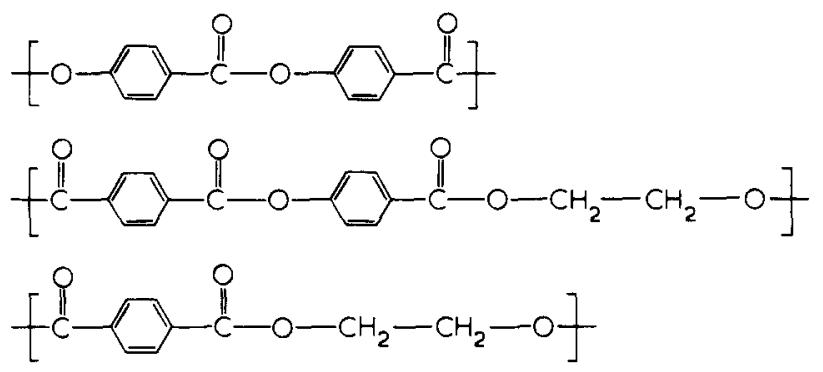

For the particular mole fraction material we have studied Jackson and Kuhfuss conclude that the segments are arranged randomly along the chain; this conclusion was drawn mainly from their n.m.r. data. The absence of any melting endotherm and the lack of any sharp wideangle $\mathrm{X}$-ray peak suggests that the material appears to behave as an amorphous random copolymer with little short range ordering. The fact that the material does not crystallize on cooling offers a considerable experimental advantage in that observations made at room temperature correspond essentially to the frozen-in mesophase textures.

Jackson and Kuhfuss observed that the copolyester had additional unusual properties. Above $275^{\circ} \mathrm{C}$ the melt appeared turbid and in this temperature region the viscosity was low, being of the order $10-10^{2} \mathrm{PaS}$. They found melt extruded samples showed a considerable degree of anisotropy in terms of their mechanical properties and from these collective observations they concluded that the behaviour of the material was consistent with that of a thermotropic nematic liquid crystal.

\section{OPTICAL TEXTURES}

The optical textures we observed either in bright field illumination or between crossed polaroids were often complex, and the detailed texture might depend on variables such as sample preparation, thermal history or mechanical history. The copolyester pellets were dried under vacuum for at least $12 \mathrm{~h}$ at $150^{\circ} \mathrm{C}$. Small pieces of the pellet were then pressed between glass slides main- 


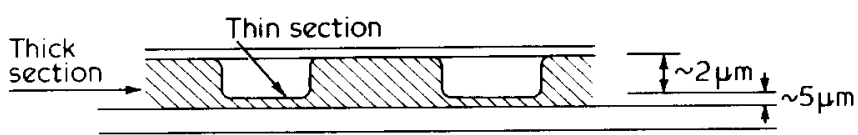

Figure 1 Schematic diagram showing the section of sample used in optical microscopy

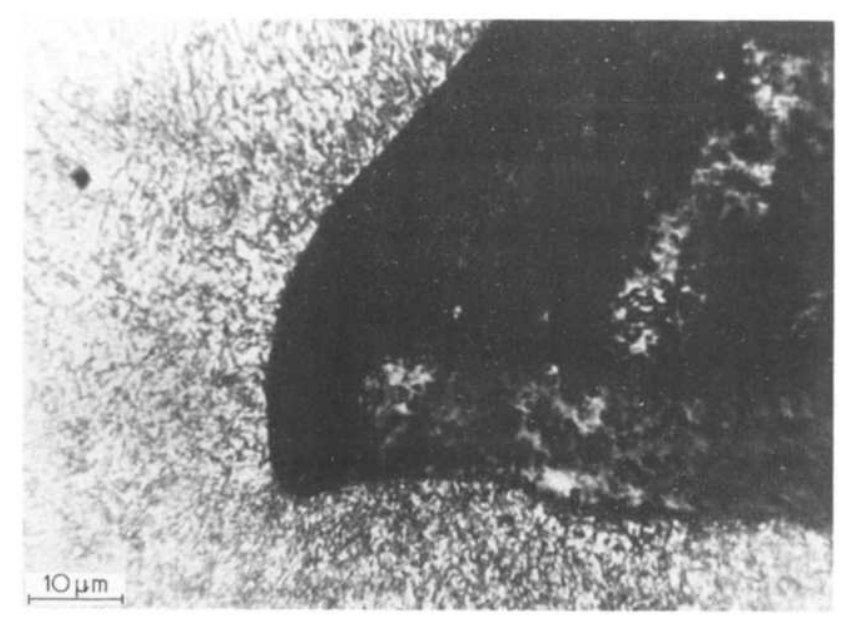

Figure 2 Typical optical micrograph viewed between crossed polaroids of sample heated to $350^{\circ} \mathrm{C}$ and cooled to room temperature. Both thick and thin sections clearly visible

tained at $220^{\circ} \mathrm{C}$; the surfaces of the glass slides had previously been washed in acetone. In order to observe transmitted light through the material it was found that the sample had to have a thickness of less than about 20 $\mu \mathrm{m}$. It was difficult to prepare samples that had uniform thickness over extended regions and usually small air or gas bubbles would form in some areas. On further heating above $220^{\circ} \mathrm{C}$ these bubbles would grow and the sample would take on the overall form shown schematically in section by Figure 1 . The 'thick section' of the sample was attached to both bottom and top glass slides. The 'thin section' was observed below bubbles in the sample, and 'talysurf measurements indicated that the thin sections were typically $5 \mu \mathrm{m}$ or less in thickness. The polymer was always observed to be attached to the bottom glass slide. We were unable to establish whether this was due to gravity, temperature gradients or effects due to the different chemical composition of the bottom glass slide and the top cover slip.

A typical room temperature optical micrograph viewed between crossed polaroids is shown in Figure 2. Here both thick and thin sections can be observed; the transition between the two regions occurs as a rapid change in sample thickness. The characteristic feature of the thick section is the existence of a complex array of dark lines observed on a light background; in addition some samples showed regions within the thick section where there was an enhanced concentration of dark lines. The texture is similar whether observed between crossed polaroids or in bright field. In contrast the texture observed in the thin section can only be observed when viewed between crossed polaroids. The general feature of the thin section consists of some areas of local birefringence where the intensity of the birefringence is dependent on the orientation of the polaroids with respect to the sample.

\section{Thick sections}

If a thick section of the form shown in Figure 2 is heated using a microscope hot stage with the sample held under a nitrogen blanket, two distinct features appear. Above about $230^{\circ} \mathrm{C}$ the sample becomes mobile and, as shown in Figure 3 for a sample heated up to $350^{\circ} \mathrm{C}$, dark threads are observed in the sample; these threads can be observed to move relatively freely through the sample. In addition to the threads, domains in the form of rings appear and the size and rate of formation of the ring domains will depend on both temperature and time. The ring domains can also be seen in Figure 3. Both rings and threads can be observed with equal clarity in bright field or between crossed polaroids. In 'small molecule' liquid crystal optical texture studies it is usual to associate threads with line singularities known as disclinations (see for example de Gennes ${ }^{5}$ ); it is probable, indeed likely, that the threads we observe in the mesomorphic copolyester are disclinations.

If the sample shown in Figure 3 is cooled to room temperature we observed that the region within the rings becomes heavily threaded as shown in Figure 4; the effect is shown in greater clarity by Figure 5. The observation that threaded material appears within the rings on cooling

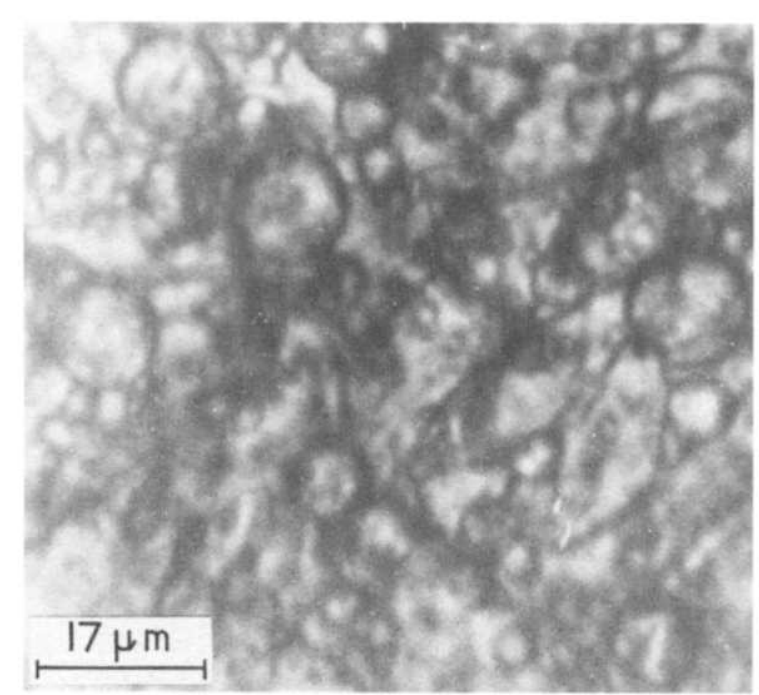

Figure 3 Rings and threads observed within a 'thick section' at $350^{\circ} \mathrm{C}$ in bright field illumination

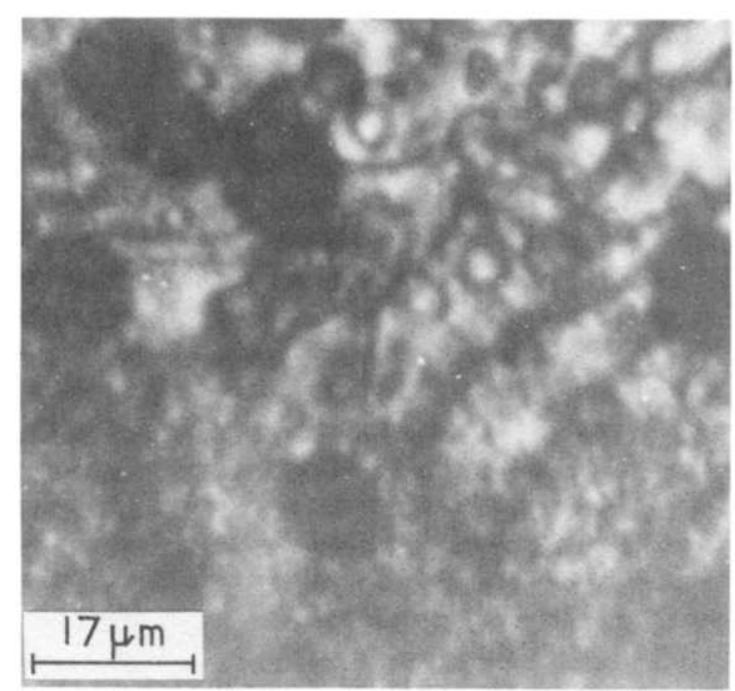

Figure 4 As Figure 3 cooled to room temperature. Darkening within rings visible 


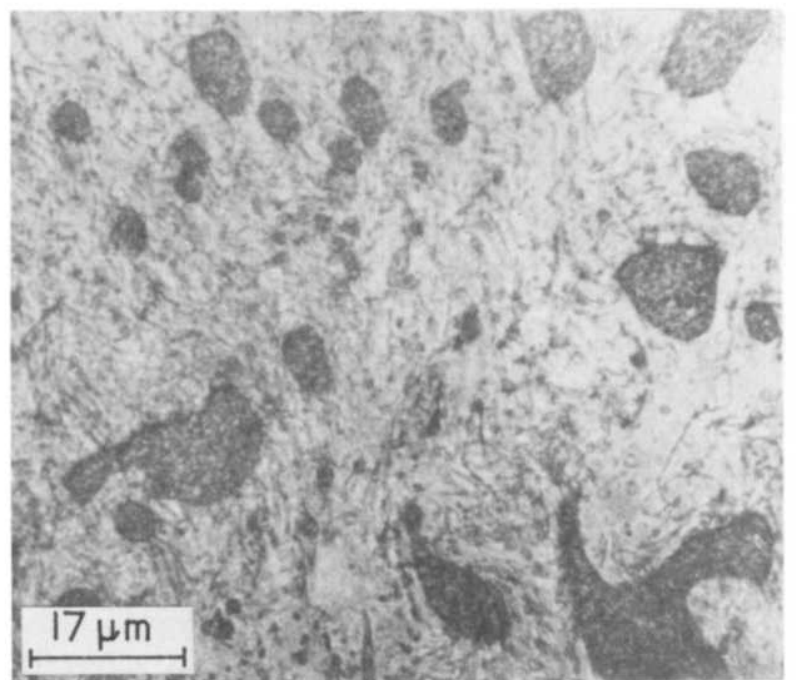

Figure 5 Heavily threaded regions within 'rings' for sample cooled to room temperature viewed between crossed polaroids

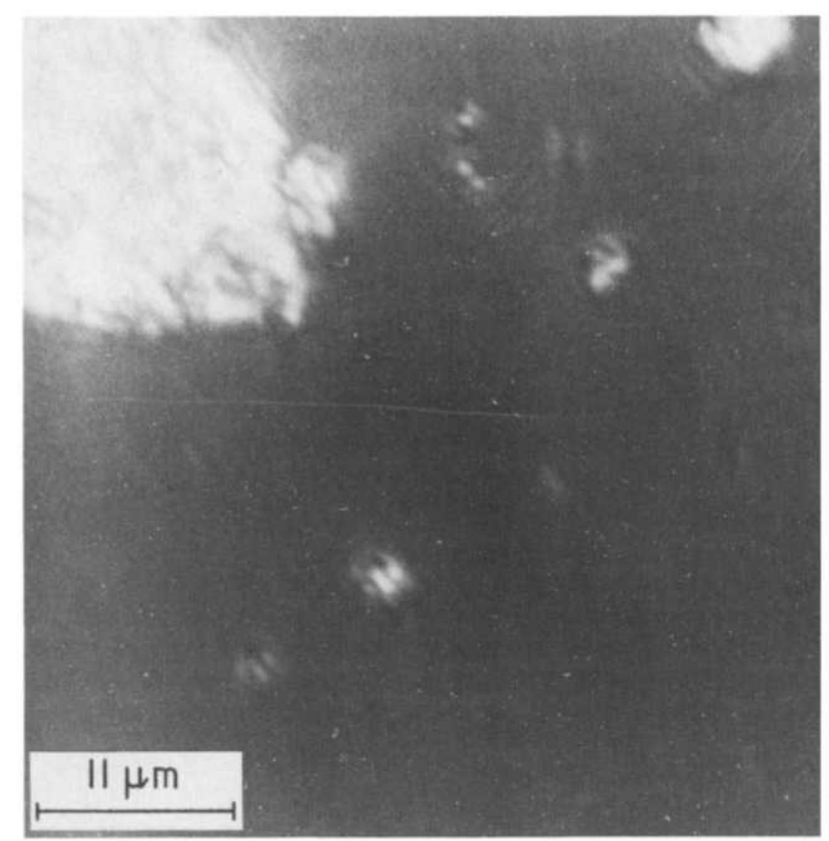

Figure 6 'Thick section' viewed between crossed polaroids at $380^{\circ} \mathrm{C}$. Isotropic and mesomorphic phase visible. Viewed between crossed polaroids

strongly suggests that the rings are not bubbles. If the material within the rings were isotropic we would expect to observe that the light intensity within the rings when viewed between crossed polaroids would decrease. As we do not see this effect we conclude that the emergence of the ring domains at elevated temperature is associated with some form of chemical or physical segregation process due to difference in chemical composition or molecular weight. As samples of fractionated copolyester were not available we were unable to establish the exact segregation process involved.

If the thick section sample was heated above $350^{\circ} \mathrm{C}$ or kept at $350 \mathrm{C}$ for times of greater than typically $10 \mathrm{~min}$ we observed regions of the sample that become dark when viewed between crossed polaroids. These dark areas remained dark on shearing the sample, thus distinguish- ing between an isotropic and homeotropic* texture. We therefore conclude that these dark regions are areas of isotropic material which can be seen in Figure 6 . The sample shown in Figure 6 was maintained at $3800^{\circ} \mathrm{C}$. However, on subsequent cooling to $350 \mathrm{C}$ the region that was previously isotropic develops a complex threaded texture as shown in Figure 7.

It would appear that refraction effects due to sudden changes in refractive index within the material have a greater effect on the observed optical contrast in thick sections than effects associated with optical anisotropy. Within the resolution of the optical microscope we have observed that these rapid changes in refractive index occur as lines, thought to be disclinations, and ring domains, thought to be associated with a segregation process that occurs at elevated temperature. We have also observed that if the temperature is sufficiently high and the time sufficiently long then regions of the material can exist in the isotropic state. As the temperature is lowered a complex mesomorphic texture is observed which on further cooling becomes frozen into the sample without further major changes.

\section{Thin sections}

In order to observe textures in thin sections it was necessary to observe the sample between crossed polaroids; the sample appeared uniformly illuminated when viewed in bright field. Figure 8 shows a series of birefringence photographs taken at $350 \mathrm{C}$ with the polaroids oriented in three different positions with respect to the sample. The observation is striking in that it clearly shows that the quiescent polymer is in an anisotropic state. Figure 9 shows the same area as Figure 8 observed at room temperature. There are slight differences in the

* Homeotropic texture, one in which the principle optical axis parallel to the symmetry axis of the molecular director is perpendicular to the surface of the sample. A homogeneous texture is where the symmetry axis of the molecular director is parallel to the surface. (See Gray for a useful discussion on liquid crystal textures and Frank ${ }^{7}$ for a discussion on the meaning of directors.)

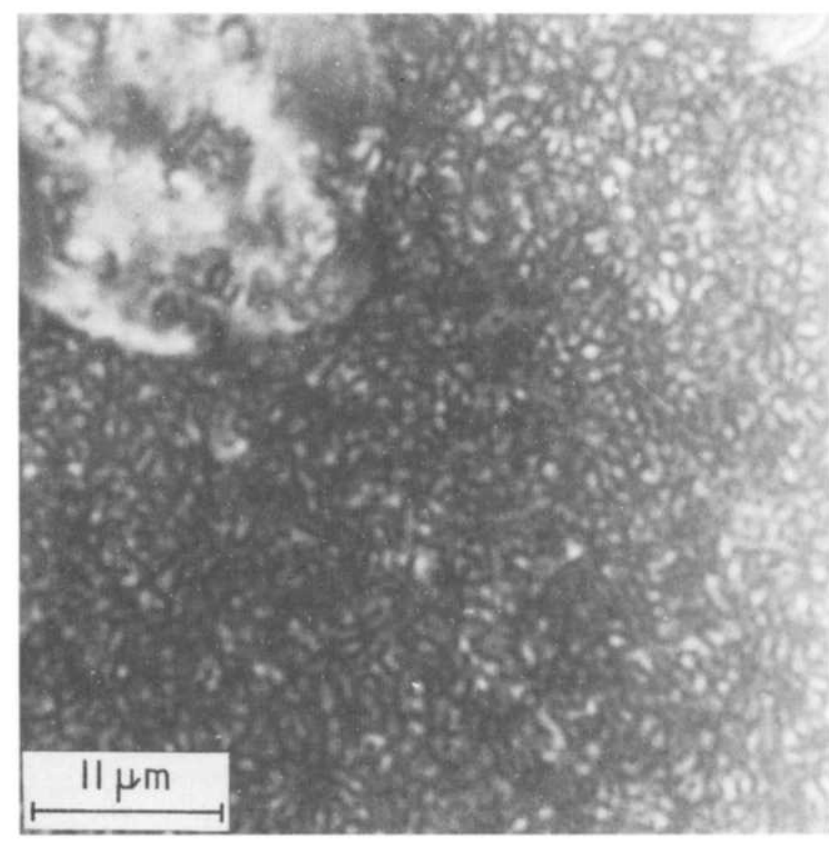

Figure 7 As Figure 6 cooled to $350^{\circ} \mathrm{C}$. Isotropic region becomes threaded 

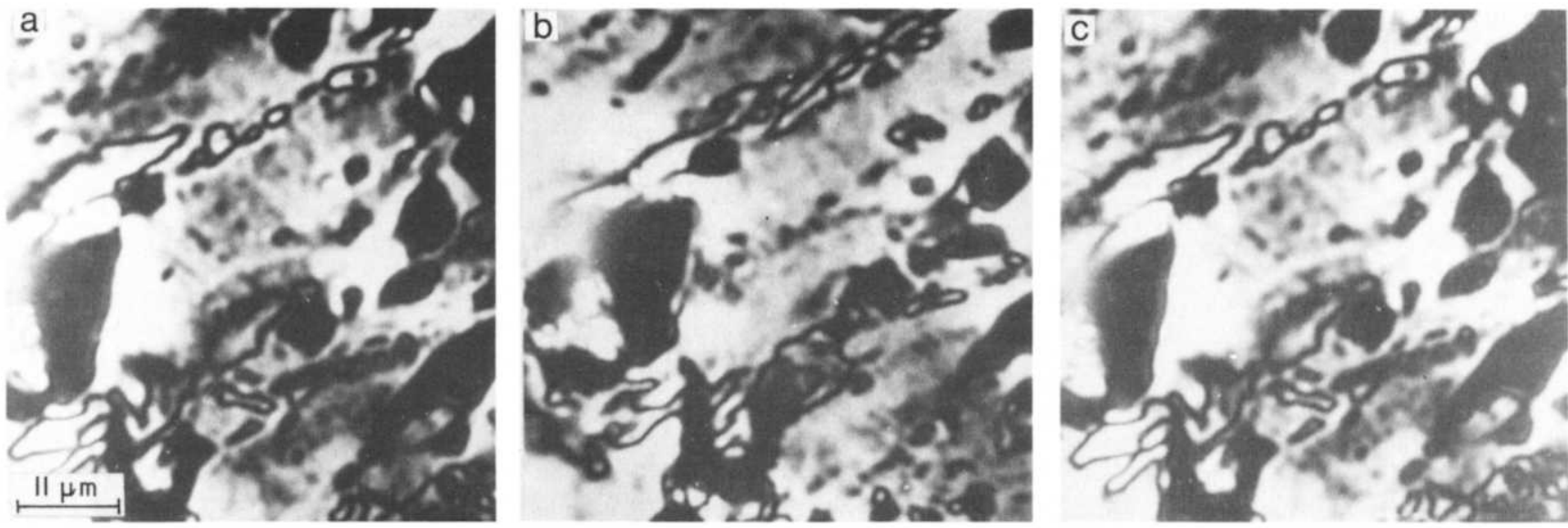

Figure 8 'Thin section' viewed between crossed polaroids at $350^{\circ} \mathrm{C}$. (a) Polarizer vertical; (b) rotated $40^{\circ}$ clockwise; (c) rotated $90^{\circ}$ clockwise
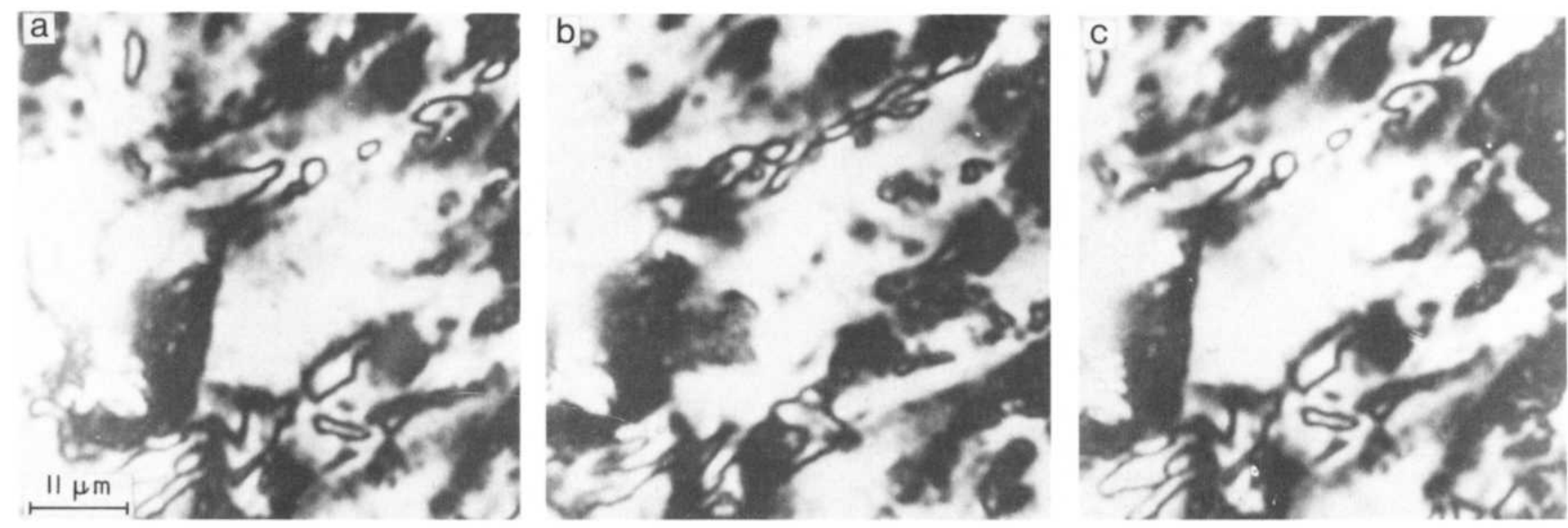

Figure 9 Same area as Figure 9 viewed between crossed polaroids at room temperature. (a) Polarizer vertical; (b) rotated 40 clockwise; (c) rotated $90^{\circ}$ clockwise
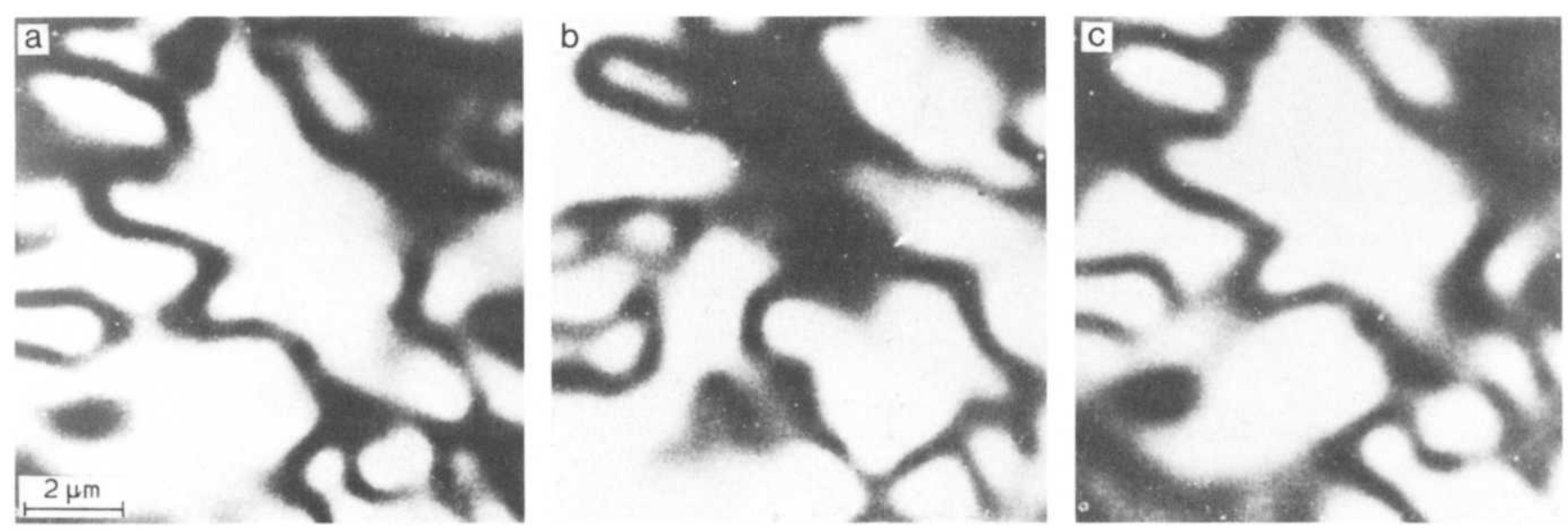

Figure 10 'Thin section', enlargement of Figure 9, homogeneous area viewed between crossed polaroids at room temperature. (a) Polarizer vertical; (b) rotated $40^{\circ}$ clockwise; (c) rotated $80^{\circ}$ clockwise

photographs but in general it can be seen that the room temperature thin section is essentially frozen in mesophase.

In either Figure 8 or 9 it can be seen that some areas remain dark for all polaroid orientations. In these areas the material is either in the homeotropic or isotropic state. We believe the former case to be true because at elevated temperatures slight shearing induced birefringence which is consistent with the occurrence of a homeotropic state.
In the remaining birefringent areas dark extinction bands are clearly seen and the position of these extinction bands is dependent on the orientation of the polaroids with respect to the sample. The magnitude of the retardation in these areas remains unchanged within our resolution of about $\lambda / 10$ and the birefringence is insensitive to focussing. In view of this we believe these areas are characterized by a homogeneous texture with the director being parallel to the surface of the glass. Figure 10 shows an enlarged 

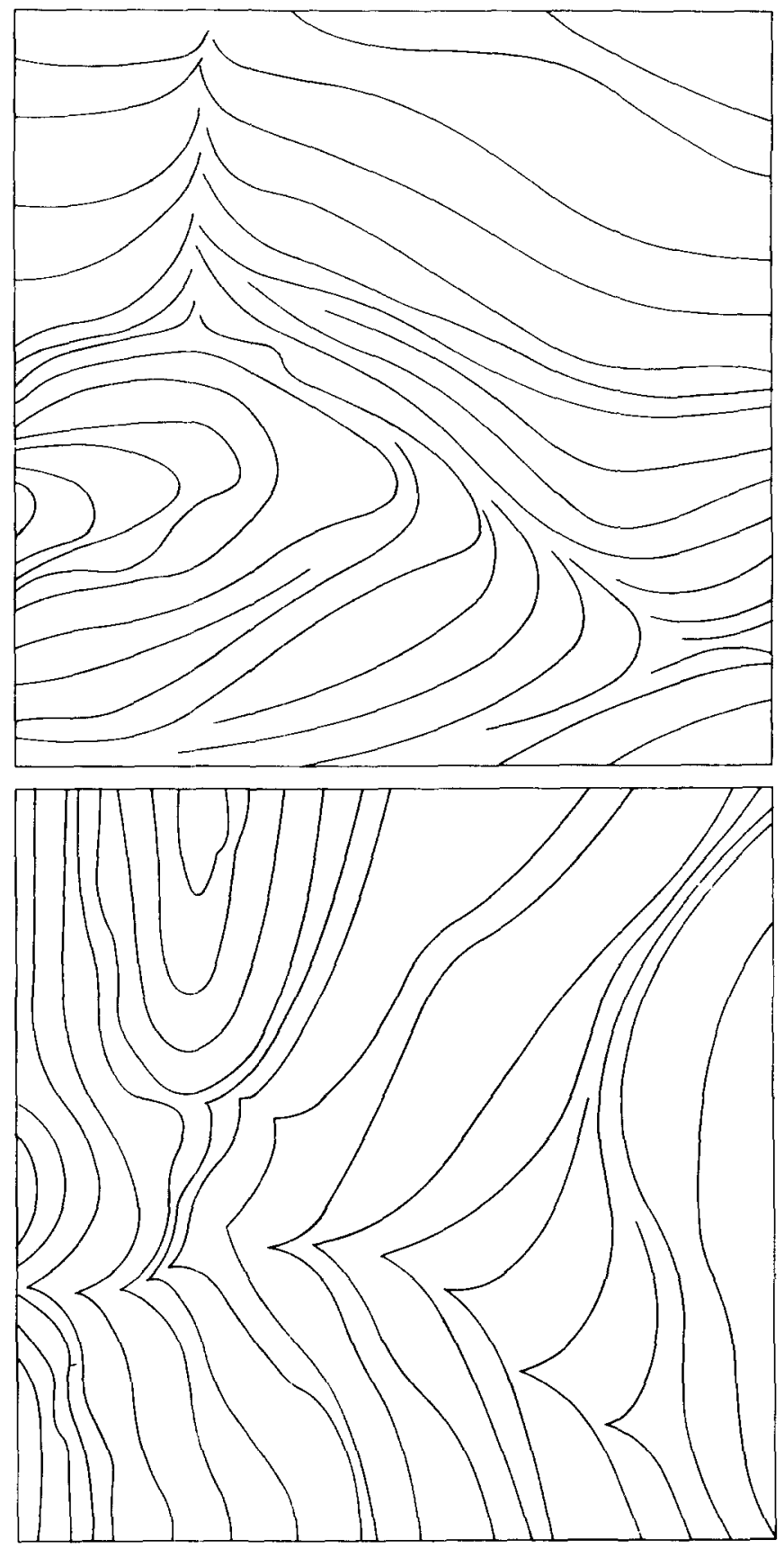

Figure 11 Schematic diagrams of molecular trajectories associated with homogeneous areas shown in Figure 10

area of this region. The three photographs are taken with the polaroids in different orientations. With the polaroids in a known orientation for regions that appear dark, the principle optical axis is either parallel or perpendicular to the polarizer or analyser. Making the assumption that the director in the region shown in Figure 10 is parallel to the surface, by varying the orientation of the polaroids and following the movement of the extinction bands it is possible to map out the 'trajectories' of the apparent optical axis. These trajectories will follow the trajectories of the director.

The map of the apparent director trajectory corresponding to the areas shown in Figure 10 is shown in Figure 11. Without knowledge of the optical signs we are unable to distinguish between the two maps that we have shown. However, it can be seen that one map is merely the orthogonal set of the other; the map was constructed from photographs taken at $10^{\prime}$ intervals. A surprising result from this analysis shows that within the apparently homogeneous area examined a domain wall exists. We believe the texture to be essentially two dimensional as the observed contrast is insensitive to focussing and the magnitude of the retardation remains constant; thus it would appear that a planar domain wall exists within the thin section. Ryschenk ow ${ }^{8}$ observed similar textures in small-molecule nematic liquid crystals.

The director map shown in Figure $1 /$ gives a clear idea of the manner in which the local orientation within the material changes. In a similar way to smallmolecule liquid crystals it is clear that the glass surface can influence the observed anisotropy of the mesomorphic polymer. It should be stressed at this point that 'normal' polymer melts would show no optical anisotropy in the quiescent state and the usual and, in general, only way in which orientation in the melt could be induced would be by means of some form of shearing flow.

Movement of bubbles and shearing in thick sections. At temperatures above about $260^{\circ} \mathrm{C}$ the gas or air bubbles present in the sample were observed occasionally to migrate within the sample. Figure 12 shows the migration of a bubble through a thick section at $380 \mathrm{C}$. The sample is viewed between crossed polaroids and several textures are present in the one photograph: the bubble seen as a ring in the photograph is migrating from left to right in the picture. Isotropic material surrounds the bubble. However, underneath the bubble and some of the isotropic material there is a 'thin section' of material attached to the bottom glass surface; this material is in the homogeneous state if birefringence is observed or homeotropic state if the area remains dark. An arrow in Figures $12 a$ and $12 b$ assigns a common landmark in each of the photographs. From Figure $12 a$ it can be seen that the movement of the bubble does not appear to affect the lower thin section material. If the temperature is reduced to $350^{\circ} \mathrm{C}$ as shown in Figure $12 b$ the material that was previously isotropic becomes birefringent and as the bubble migrates through the mesomorphic material some of the thin section material becomes birefringent suggesting that this thin section material converts from a homeotropic to non-homeotropic texture. In other areas the migration of the bubble causes homogeneous areas to become homeotropic. In general the textures will return to their original configuration in a few seconds as the bubble passes.

The shearing behaviour of the thin section near the surface is perhaps more clearly demonstrated in Figure 13 which shows the shearing of an edge of a thick section over a thin section. The sequence of photographs was taken at $350 \mathrm{C}$. In Figure $13 a$ the thick section seen as the opaque material in the upper half of the photograph has been sheared vertically with respect to the photograph. The edge of the thick section has moved upwards and as it moved induced birefringence in the homeotropic thin section on the hottom surface. With time this birefringence decays. The time sequence shows that a relaxation time of order $10 \mathrm{~s}$ is representative of the decay in orientation. The photographs were taken at $350 \mathrm{C}$ which indicates that overall relaxation behaviour of this mesomorphic polymer can be expected to be considerably longer than conventional polymer melts.

\section{DIRECT OBSERVATIONS OF DISCLINATIONS}

Conventional polymer melts can normally only be oriented by an external shearing flow. Most liquid crystals are 
Mesomorphic copolyester: M. R. Mackley et al.
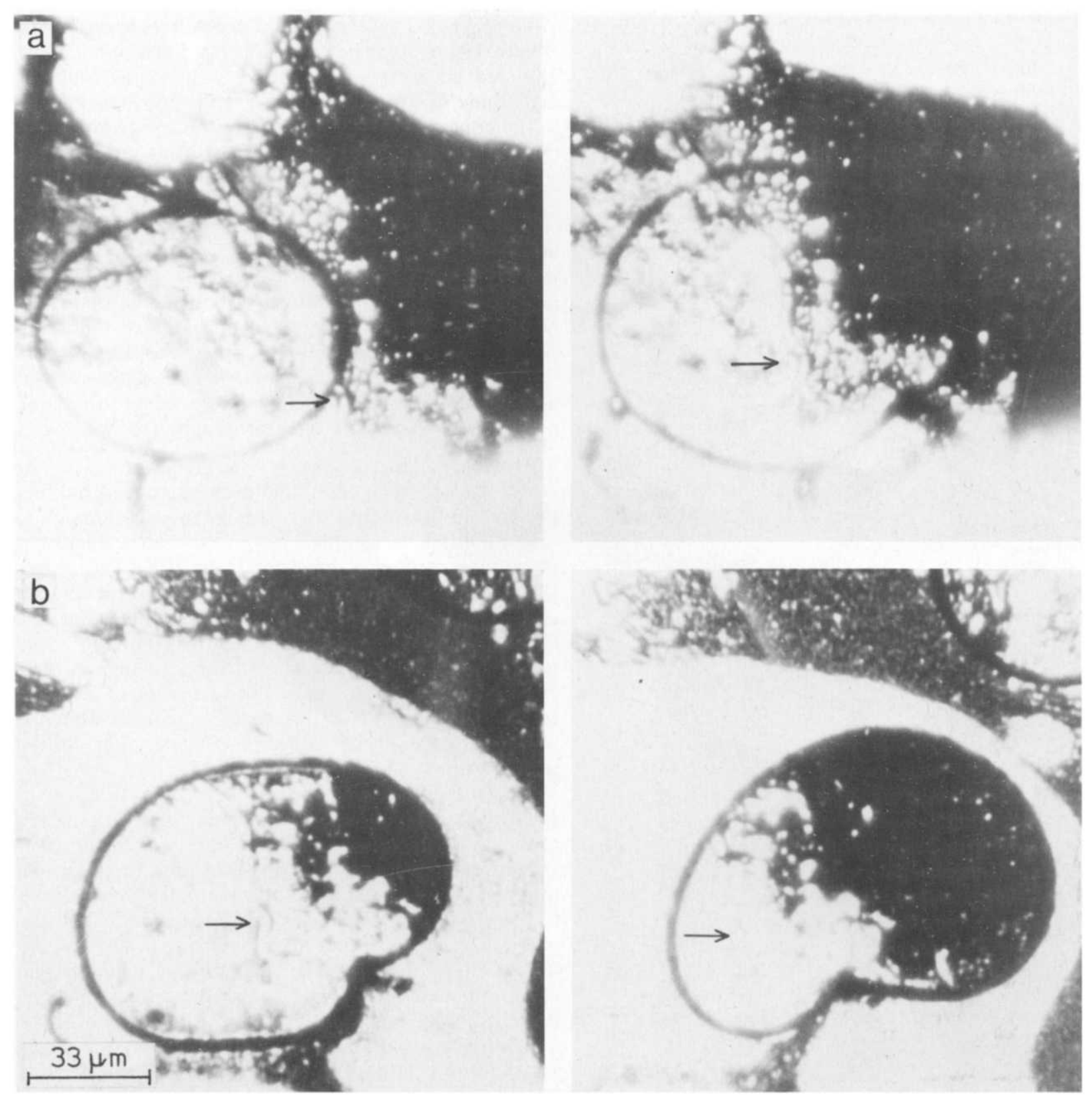

Figure 12 Migration of bubble within thick section, viewed between crossed polaroids at: (a) $380^{\circ} \mathrm{C}$; (b) $350^{\circ} \mathrm{C}$
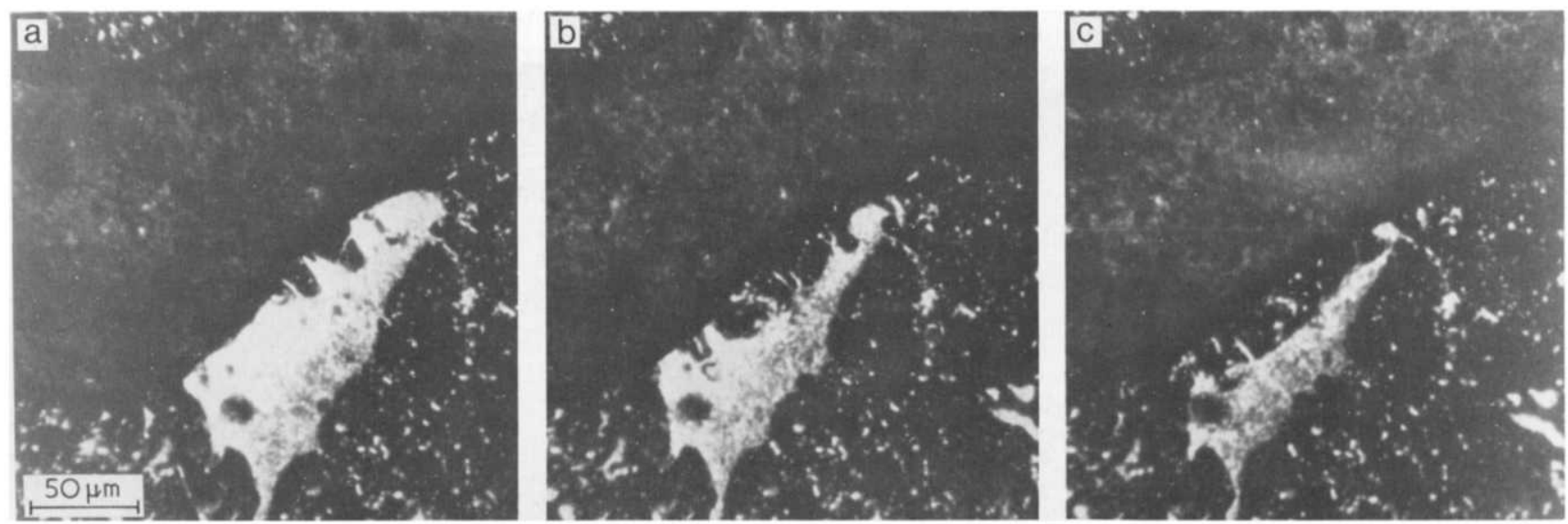

Figure 13 Shearing of 'thick section' over top of 'thin section', observed at $350^{\circ} \mathrm{C}$ between crossed polaroids: (a) time $t=1 \mathrm{~s}$ after shearing; (b) $t=3 \mathrm{~s} ;$; $(\mathrm{c}) \mathrm{t}=6 \mathrm{~s}$ 
sensitive to flow, surfaces, electric and magnetic fields and with this in mind we examined the behaviour of the copolyester in a magnetic field.

The samples to be tested were prepared between glass slides in the manner previously described. The glass slide was then placed in a small cylindrical furnace that in turn was positioned between the pole pieces of an electromagnet. A field of $7 \mathrm{k}$ Gauss was applied either parallel or perpendicular to the surface of the glass slide. The sample was then heated up to a temperature of $320 \mathrm{C}$ which took approximately $5 \mathrm{~min}$. After holding at $320 \mathrm{C}$

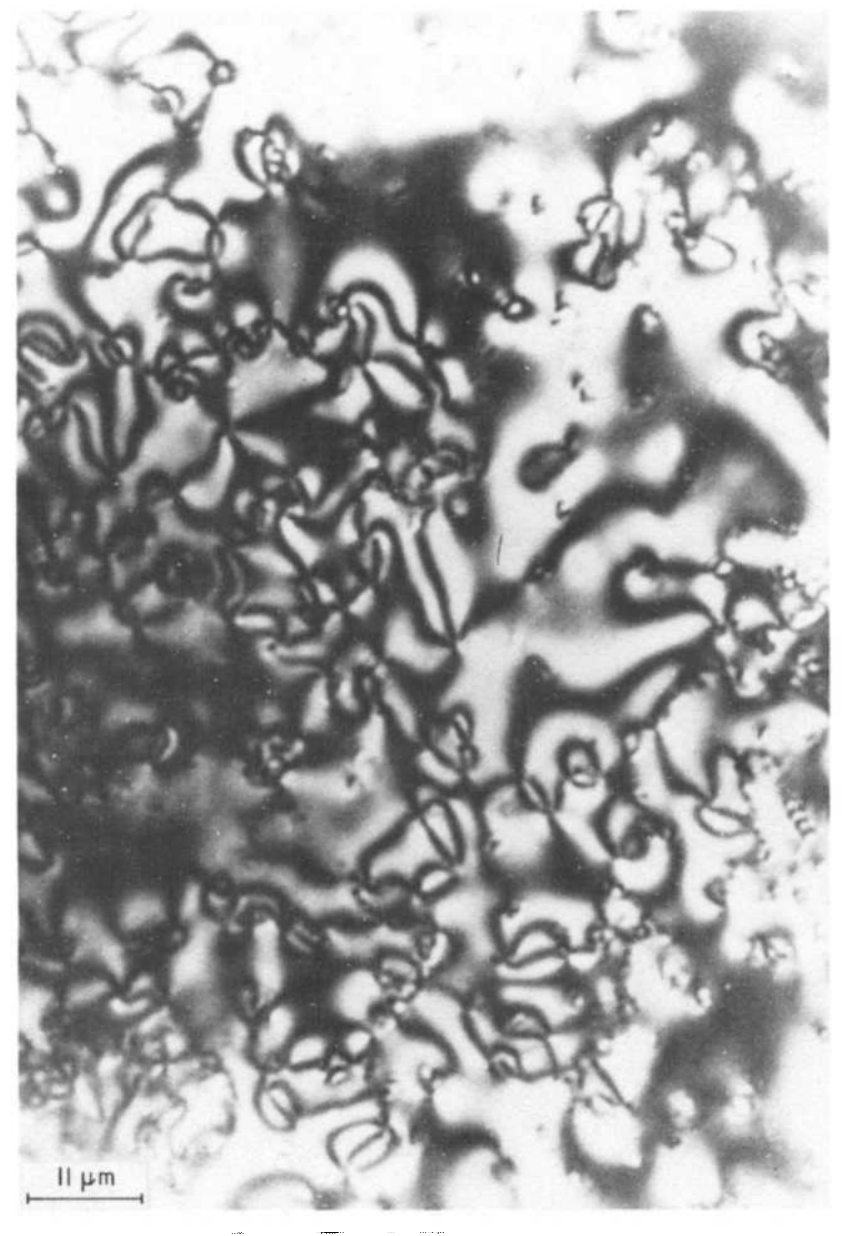

Figure 14 Effect of magnetic field. Field of $7 k$ Gauss applied parallel to surface of glass slide. 'Thick section' viewed between crossed polaroids with polarizer vertical for 5 min the sample was allowed to cool with the magnetic field remaining on. Cooling to $100 \mathrm{C}$ took typically $15 \mathrm{~min}$.

Unfortunately our observed findings were inconclusive concerning the effect that magnetic field has on the alignment of the material under the above conditions. Some of our early experiments showed interesting textures which we include here because they show direct evidence for the existence of disclinations in the material When the experiment was subsequently repeated we were unable to obtain the initial textures observed and the material showed little or no influence from the application of magnetic field.

Some of the optical textures we observed are shown in Figures 14, 15 and 17; the thickness of the sample corresponds to what we have previously called thick sections. The texture seen in Figure 14 is different from anything previously observed for this material and bears a striking resemblance to textures observed in smallmolecule nematic liquid crystals showing the presence of disclinations (see for example Chandrasekhar ${ }^{10}$ ). The area shown in Figure 14 is consistent with the material being in a homogeneous form with a large number of centre textures present. Figure 15 shows clearly the way in which the extinction bands move with respect to the local rotation symmetry axis at each centre. In all cases four extinction bands are seen to radiate from each centre and approximately equal numbers of extinction bands rotate clockwise or anticlockwise as the polaroids are rotated.

We can interpret the optical anisotropy associated with these centred textures in terms of disclinations that run vertically from the top to the bottom glass slide. In order to identify positively the existence of disclinations optically it is necessary to observe the disclination in the geometric orientation corresponding to Figures 14 and 15.

Frank $^{4}$ has classified the types of disclinations that can be expected in a two dimensional ordered nematic liquid crystal. The optical behaviour of our centre textures are consistent with disclinations of strength +1 and -1 following the normal definition of disclination strength (see for example Chandrasekhar ${ }^{10}$ ). The trajectories of the directors in the region near a disclination were given by Frank ${ }^{4}$ and these are shown for \pm 1 disclinations in Figure 16. From our observations we are unable to identify which of the three possible +1 disclinations were present.
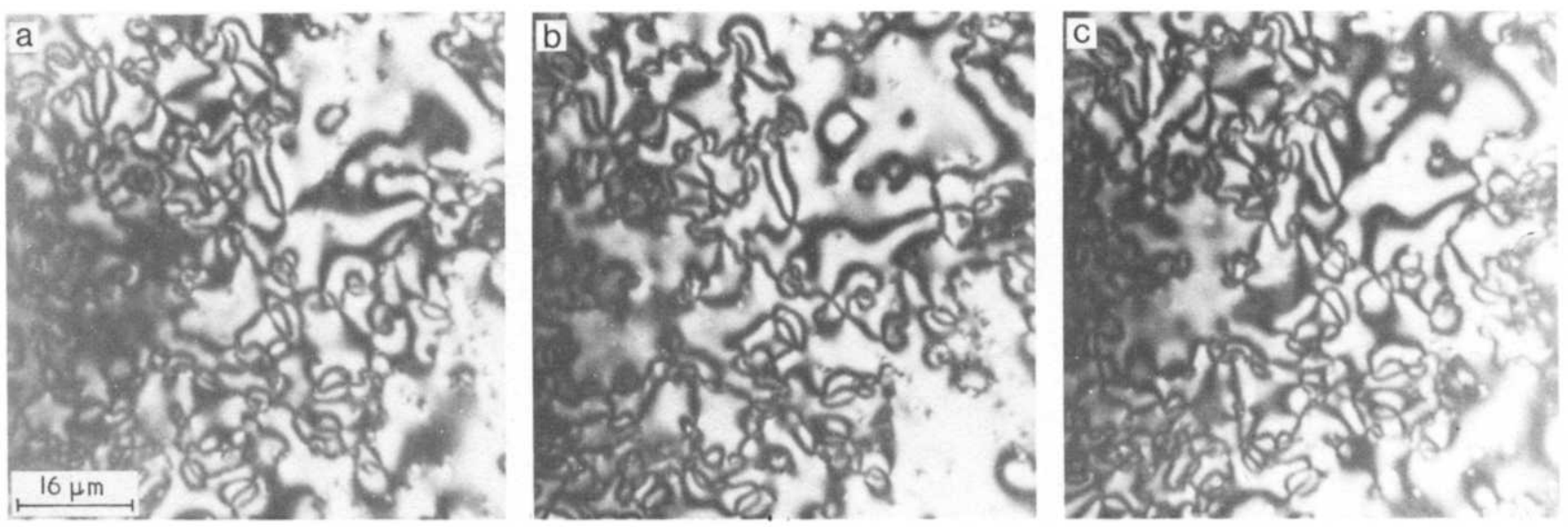

Figure 15 The effect of magnetic field. As Figure 14 with polarizer rotated: (a) $10^{\circ}$ clockwise; (b) $40^{\circ}$ clockwise; (c) $70^{\circ}$ clockwise 


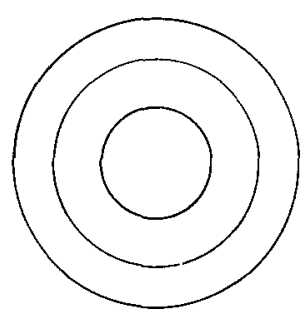

$+1$

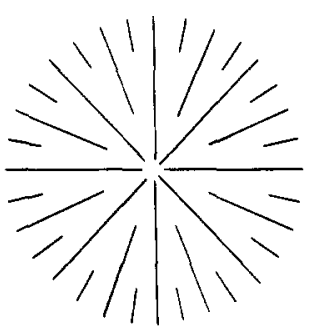

$+1$
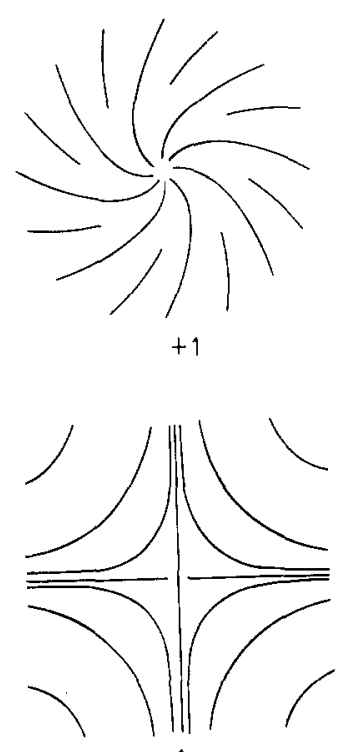

$-1$
Figure 16 Schematic diagram of molecular trajectories associated with disclinations with sign \pm 1

In the early experiments, when the magnetic field was applied at right angles to the surface of the slide a texture of the form shown on Figure 17 was observed. The difference in comparison with Figure 14 is marked. We believe that the dark lines seen in Figure 17 in both crossed polaroids and in bright field correspond to disclinations viewed from the 'side' rather than in Figure 14 where they are viewed along the axis of the disclination.

The textures we have reported in this section confirm the existence of disclinations of type \pm 1 within the material. The fact that we at present are unable to reproduce these experiments raises doubts as to the effect of magnetic field alone in obtaining this texture. We have established that surfaces can influence this material and together with other factors such as chemical purity, pressure, thermal and mechanical history it is now clear from our subsequent experiments that the centre textures could have resulted from a combination of any of these or other influences.

\section{ELECTRON MICROSCOPY}

We examined two representative samples of the bulk copolyester. The first sample was the as received extruded pellet; this material had an overall anisotropy associated with orientation along the extrusion direction. We found great difficulty in preparing a bulk sample that showed no resultant preferred orientation. However, a reasonable approximation to this state was achieved by heating a pellet of the material encapsulated in metal foil and heated under vacuum to a temperature of $350^{\circ} \mathrm{C}$; holding for a period of $3 \mathrm{~min}$ and subsequent cooling to room temperature over a period of a few minutes. It should be stressed in these and all other elevated temperature experiments, problems associated with degradation could be severe unless suitable precautions were taken.

In order to examine the interior of the material, samples were fractured using a micro three point bending device. It was found necessary to perform the fracture at liquid nitrogen temperature in order to obtain a brittle fracture with apparently no observed plastic deformation associated with the fracture process.
Scanning electron micrographs of the two fracture surfaces associated with each sample are shown in Figures 18 and 19 . The fracture surface of the anisotropic sample in Figure 18 shows a well-oriented micaceous structure, the surface bears a striking resemblance to the fracture surfaces of some insect cuticles such as locust legs; in these cases the basic building material is the natural polymer chitin which is also known to form liquid crystal types of organization (Neville ${ }^{11}$ ). The fracture surface of the 'isotropic' sample shown in Figure 19 appears smooth and does not show any of the detailed structure shown in Figure 18.

We are unable to prepare reliable cut thin section samples suitable for transmission electron microscopy; however, we were able to prepare surface replicas. The preparation sequence consisted of evaporating a thin coating of carbon onto the surface to be examined. The carbon film was then shadowed with gold and the polymer substrate subsequently removed by immersing the whole sample in the solvent $o$-chlorophenol; this left a thin shadowed carbon film suitable for transmission electron microscopy.

In order to establish the effect of shadowing alone, a clean glass slide was carbon coated and then shadowed and the resulting effect is shown in Figure 20. Surface replicas of the fractured anisotropic sample shown in Figure 21 revealed little additional information. The only characteristic feature observed was the occurrence of steps on the surface; typical depths of the steps were observed to be a few $\mu \mathrm{m}$ and these observations were consistent with the scanning electron micrographs ob-

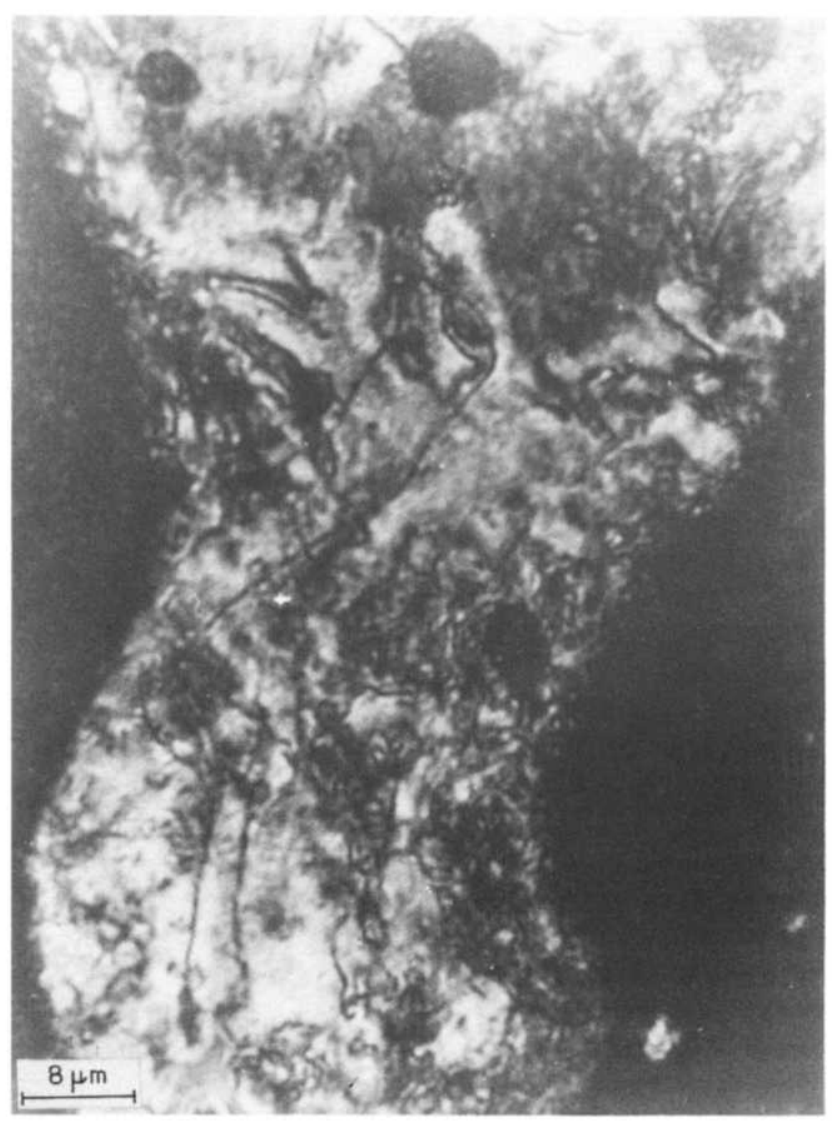

Figure 17 The effect of magnetic field. Field of $7 \mathrm{kGauss}$ applied perpendicular to surface of glass slide. 'Thick section' viewed between crossed polaroids 


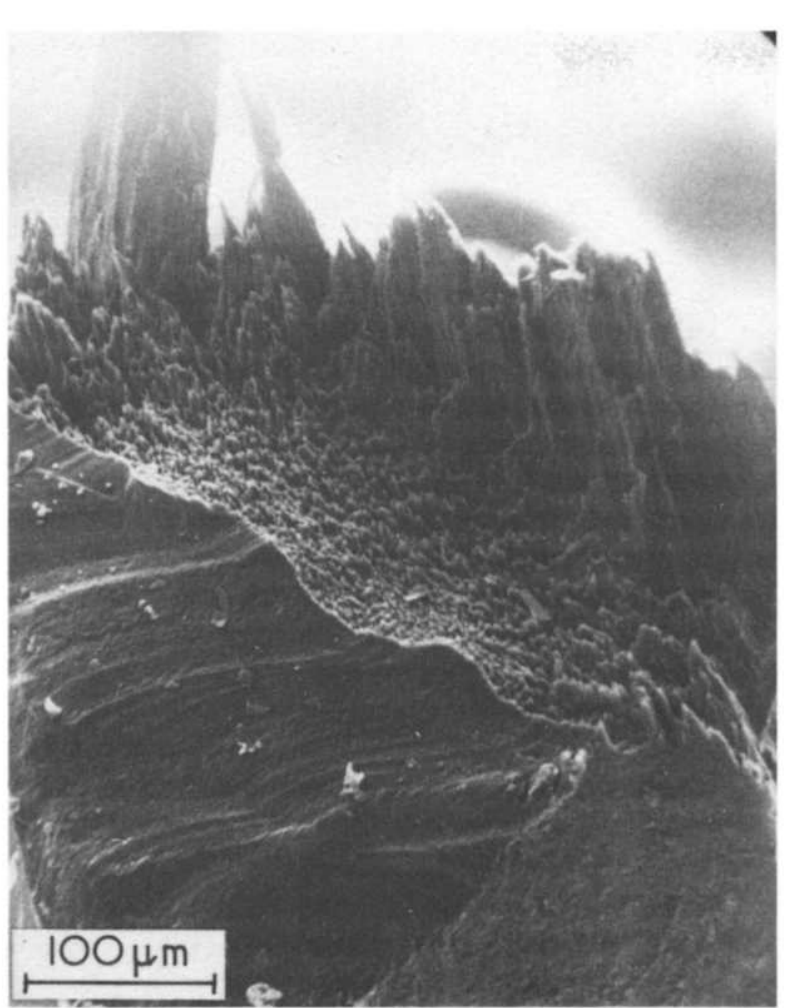

Figure 18 Scanning electron micrograph of low temperature fracture surface for extruded sample

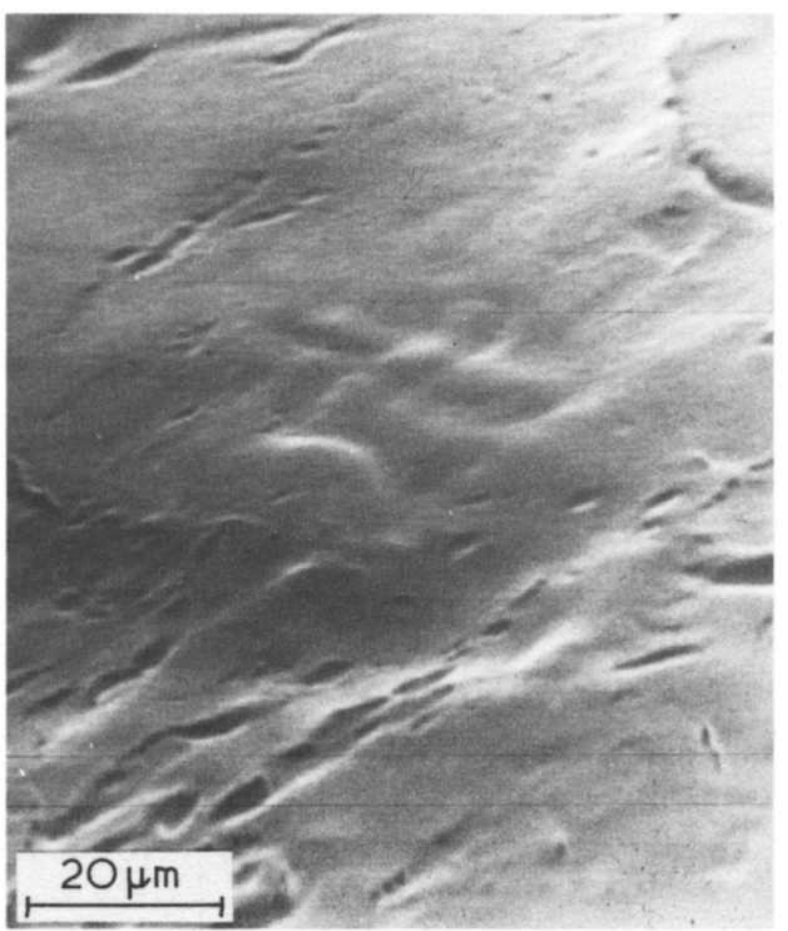

Figure 19 Scanning electron micrograph of low temperature fracture surface for 'isotropic sample'

tained for this material. Figures 22 and 23 show transmission electron micrographs of the sample that has little, if any, overall bulk anisotropy. The scanning micrograph fracture surface showed little detail; however, at the high resolution of the transmission electron microscope a characteristic and reproducible texture is observed. Comparison with Fiqure 20 allows the shadowing effect alone to be taken into account. This leaves a texture which has the feature of elongated entities oriented at different directions to the surface under examination. Because we are looking at a surface replica the observed contrast will be a consequence of surface topography, it is clear from the micrographs that there is an apparent lateral ordering with a spacing of typically $5-10 \mathrm{~nm}$. It is tempting to envisage that the mesomorphic polymer itself possesses this lateral ordering which extends over large regions. It would appear from micrographs such as Figure 20) that the orientation of these elongated entities can change over short distances and in addition it is tempting to relate these sudden changes in orientation with the domain walls that we observed optically in the 'thin section' material and illustrated schematically in Fiqure 11 . Our complete molecular trajectory map shown in Figure 11 bears a striking resemblance to the micrographs in Figures 22 and 23 although the dimension scales are somewhat different.

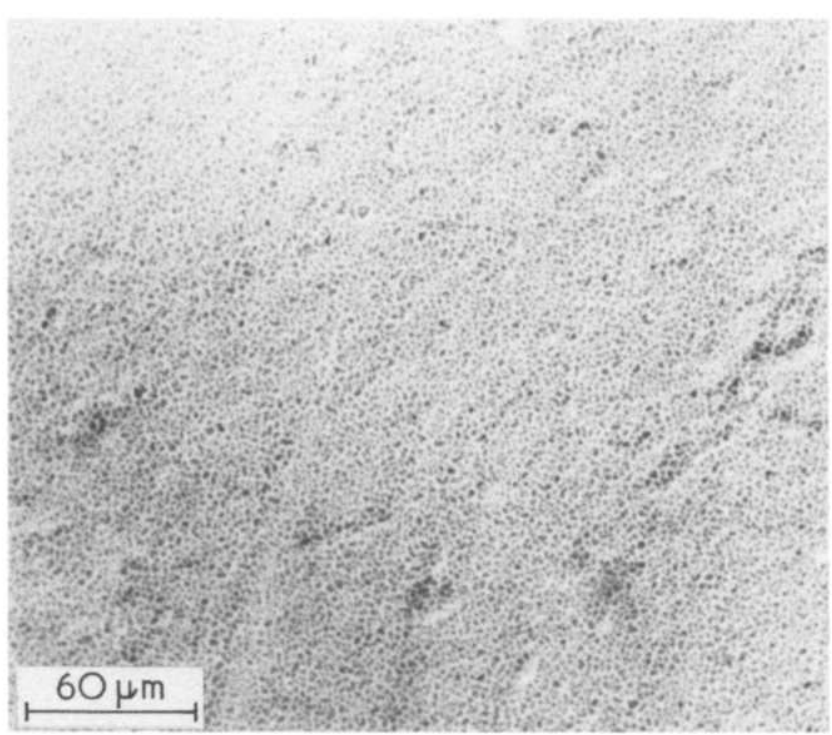

Figure 20 Transmission electron micrograph of a carbon substrate shadowed with gold

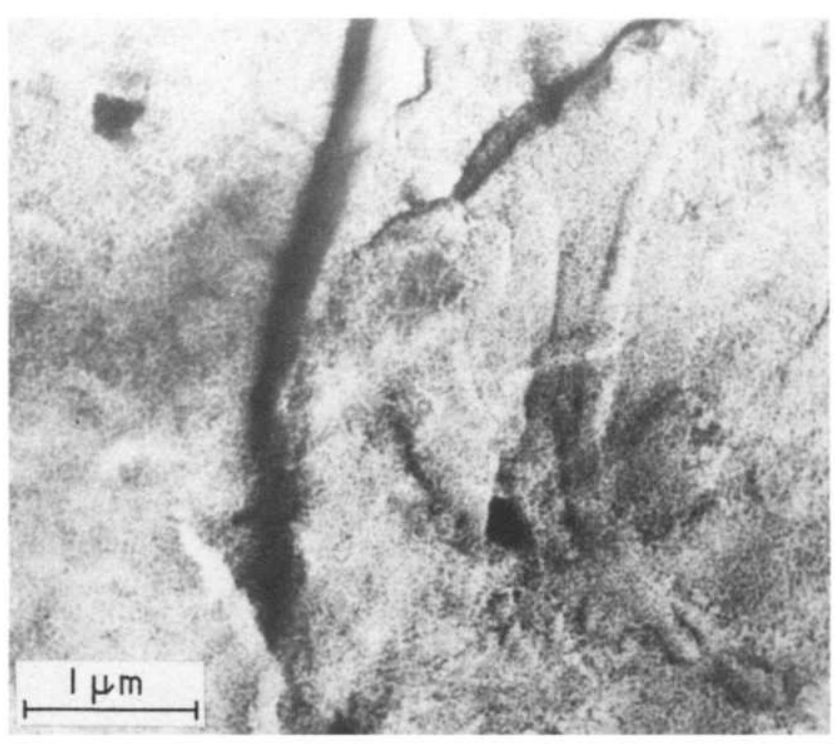

Figure 21 Transmission electron micrograph of shadowed surface replica for the low temperature fracture surface of the extruded sample 

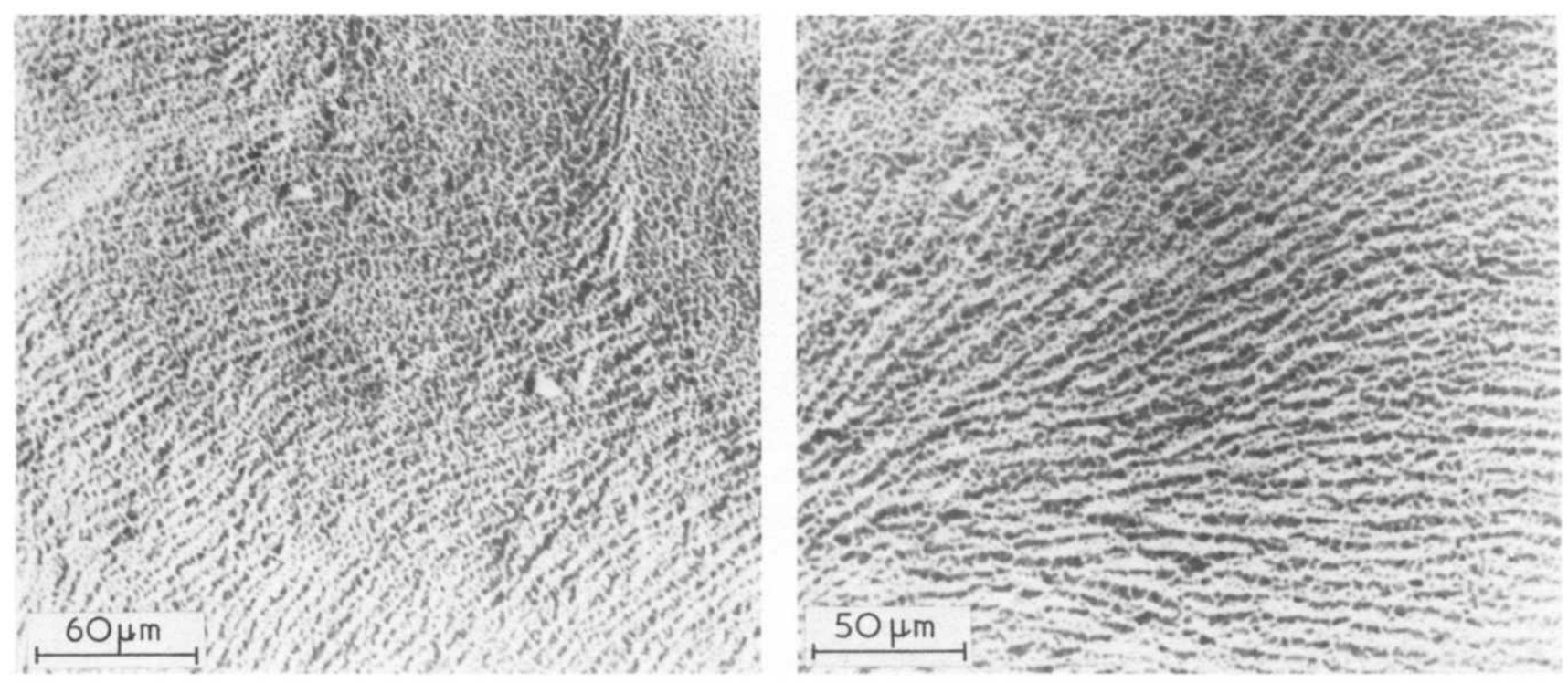

Figures 22 and 23 Transmission electron micrograph of shadowed surface replica for the low temperature fracture surface of the 'isotropic sample'

\section{CONCLUSIONS}

It is clear from our limited observations that the physical organization of the copolyester we examined is exceedingly complex. Unlike conventional polymers the material can be intrinsically ansiotropic, also we have shown that the material responds to other external fields in addition to flow. It is our objective in future work to establish the mechanisms by which the overall anisotropy of the sample is derived by the shearing of the locally anisotropic material.

\section{ACKNOWLEDGEMENTS}

We wish to thank Rhône Poulenc (France) for their financial support of the work and Eastman Kodak Corporation (USA) for their generosity in supplying us with the copolyester. M. R. Mackley wishes to thank Professor R. W. Cahn for his encouragement and support of the project and also wishes to thank Dr A. T. Simpson for the kind load of his magnet.

\section{REFERENCES}

Onsager, L. Ann. NY Acad. Sci. 1949, 52,627

Flory, P. J. Proc. Roy. Soc. London (A) 1956, 234, 73

Morgan, P. W. Macromolecules 1977, 10, 1381

Jackson, W. J. and Kuhfuss, H. F. J. Polym. Sci. 1976, 14, 2043 de Gennes, P. G. 'The Physics of Liquid Crystals' Clarendon, 1974

6 Gray, G. W. 'Molecular structure and properties of liquid crystals' Academic Press, 1962

7 Frank, F. C. 'Int. Conf. Liquid Crystals' Bangalore Dec. 3-9 1979 (Ed. S. Chandrasekhar) Heydon, London, 1980

8 Ryschenkow, G. and Kleman, M. J. Chem. Phys. 1976, 64, 404

9 Frank, F. C. Discuss. Faraday Soc. 1958, 19-28

10 Chandrasekhar, S. 'Liquid Crystals' Cambridge University Press, 1977

11 Neville, A. C. 'Structure of fibrous biopolymers', (Eds. E. D. T. Atkins \& A. Keller) Colston Soc., Butterworths, 1975 